\title{
The role of diabetes self-care education and practice in the management of type 2 diabetes mellitus (T2DM)
}

\author{
OLUSEGUN ADAMS ${ }^{1, A-D, F}$, OLABODE M. SHABI 1, A, D, F, BUSUYI KOLADE AKINOLA ${ }^{2, \text { B, D-F }}$ \\ ORCID ID: 0000-0003-4345-796X \\ OLUSEGUN E. GABRIEL ${ }^{1, A, D}$, F, IBRAHIM S. BELLO ${ }^{3, A, c, ~ D, ~ O L O L A D E ~ A K I N P E L U ~}{ }^{4, B-D, G}$
}

\author{
${ }^{1}$ Department of Family Medicine, Federal Teaching Hospital, Ido-Ekiti, Ekiti State, Nigeria \\ ${ }^{2}$ Department of Human Anatomy, Federal University of Technology Akure, Nigeria \\ ${ }^{3}$ Department of Family Medicine, Obafemi Awolowo University Teaching Hospital, lle Ife, Osun State, Nigeria \\ ${ }^{4}$ Department of Pharmacology, Obafemi Awolowo University, Ile Ife, Osun State, Nigeria
}

A - Study Design, B - Data Collection, C - Statistical Analysis, D - Data Interpretation, E - Manuscript Preparation, F - Literature Search, G - Funds Collection

Summary Background. Diabetes self-care is yet to be incorporated into diabetes management policy, hence the need to establish its practice as an essential catalyst for good glycemic control in the management of diabetes patients.

Objectives. This study was aimed at determining the role of diabetes self-care education (DSME) and practice in the management of T2DM patients.

Material and methods. A hospital-based study was conducted on 120 adults with T2DM who had been presenting at the clinic for at least two months. The study participants were divided into two groups. Group 1 was the test group and had DSME, while group 2 served as the control. Data was collected using a questionnaire, and clinical parameters of glycemic control were measured both preand post-intervention period.

Results. A significant relationship was found between diabetes education, diabetes self-care and glycemic control. This was demonstrated by the significant difference in the proportion of those who achieved good FPG from $28.3 \%$ pre-intervention to $51.7 \%$ post-intervention ( $p=0.001$ ); $2 \mathrm{HPP}$ from $30 \%$ to $46.7 \%(p=0.060)$ and $\mathrm{HbA}_{1 \mathrm{c}}$ from $53.3 \%$ to $85 \%(p<0.001)$ in the test group; while that of the control was FPG $18.3 \%$ to $30 \%(p=1.355)$, 2 HPP $11.7 \%$ to $26.7 \%(p=0.369)$ and $\mathrm{HbA}_{1 \mathrm{c}} 43.3 \%$ to $53.3 \%(p=0.804)$. This showed a significant improvement in the glycemic control of the test group compared to the control group.

Conclusions. We conclude that good knowledge and practice of diabetes self-care is relevant to the achievement of good glycemic control.

Key words: diabetes mellitus, self care, glycemic control, blood glucose, glycated hemoglobin A.

Adams O, Shabi OM, Akinola BK, Gabriel OE, Bello IS, Akinpelu O. The role of diabetes self-care education and practice in the management of type 2 diabetes mellitus (T2DM). Fam Med Prim Care Rev 2021; 23(4): 391-399, doi: https://doi.org/10.5114/fmpcr.2021.110350.

\section{Background}

Diabetes mellitus (DM) is a chronic, non-communicable disease with an increasing prevalence worldwide [1]. It constitutes a significant health and socio-economic burden for patients and healthcare systems [2]. The four main types of non-communicable diseases are cardiovascular diseases, cancers, chronic respiratory diseases and diabetes [3]. Their treatment is usually expensive and long term, thus threatening patient's budgets and putting them at high risk of poverty [4].

It was estimated in 2015 that more than $60 \%$ of all deaths were due to chronic, non-communicable diseases [3], with 415 million people worldwide suffering from diabetes and five million dying due to the disease [5]. By 2040, it has been estimated that the number of people with diabetes will rise to 642 million [5]. More than $78 \%$ of the diabetes burden will be in the low and medium income earning countries by 2020 [5]. Most people feel overwhelmed by the continuous burden of the disease and its management [6]. These emotional and social burdens may be compounded by the acute physical distress of hypoglycemia or hyperglycemia and by the chronic physical distress of diabetes-related complications [7]. In the management of diabetes, glucose control is almost entirely in the hands of the patient, and their motivation to eat, exercise, take medication, test glu- cose levels and maintain a normal body weight all contend with life's other motivations [8]. Self-care, which is an example of the Patient-Centered Care Method (PCCM), is an important aspect of living with chronic disease. It is innovative health care that is grounded in a mutually beneficial partnership among healthcare providers, patients and families. It applies to patients of all ages and may be practiced in any healthcare setting [9].

Diabetes self-care (DSC) activities or behaviors are undertaken by people with or at risk of diabetes in order to successfully manage the disease on their own under the guidance of their healthcare provider [10]. According to the American Diabetes Association (ADA), these are essential self-care behaviors in people with diabetes which predict good outcomes [11]. They include healthy eating, being physically active, monitoring blood glucose, compliance with medications, good problem-solving skills, healthy coping skills and risk-reduction behavior [10].

It is well established that good glycemic control and control of other cardiovascular risks in diabetic patients leads to reduced morbidity and mortality and improve quality of life [11-13]. The introduction of home blood glucose monitors in the 1980s and the use of glycosylated hemoglobin as an indicator of metabolic control (which was recommended by World Health Organization (WHO) in 2011) have also contributed to changes in the approach to diabetes self-care and thus have shifted more responsibility to the patient $[13,14]$. The challenge for the pa- 
tients and their relations is how to obtain the necessary skills to effectively manage their diabetes.

\section{Objectives}

The aim of the research was to evaluate the influence of diabetes self-care education and practice on the glycemic control of type 2 diabetes patients.

\section{Material and methods}

\section{Study area}

The study was carried out in the outpatient clinic of the Department of Family Medicine, Federal Medical Centre, Ido-Ekiti. This center is a Federal Government-owned 169-bedded tertiary health institution established in the year 1998. The facility is located in Ido-Ekiti, the semi-urban headquarters of Ido-Osi local government area of Ekiti State, Nigeria. The Department of Family Medicine at the center provides primary and secondary care to all categories of patients on an in- and out-patient basis. The out-patient clinic runs for 5 days a week (Monday to Friday), while, the in-patient service runs 24 hours, 7 days a week.

\section{Study design}

This was a hospital-based interventional study. All persons with type 2 diabetes attending the General Outpatient Clinic of the Federal Medical Center, Ido-Ekiti, whose ages were between 30-80 years and had been receiving treatment for at least two months at the hospital were included in this study. A questionnaire and other relevant instruments were used to collect data.

Inclusion criteria:

1. Patients aged $30-80$ years,

2. Patients diagnosed as having T2DM,

3. T2DM patients that had been receiving treatment for at least two (2) months in the hospital.

Exclusion criteria:

4. Diabetic patients that are insulin dependent,

5. Critically ill diabetic patients,

6. Diabetic patients with co-morbid factors that affect self-care, such as diabetic foot syndrome, hemoglobinopathies and other debilitating conditions.

\section{Research protocol}

The study was conducted in two phases. The purpose and benefits of the study was explained to the participants.

\section{Recruitment of participants}

392 patients were encountered within the period of recruitment. 10 respondents were excluded, while 7 did not give their consent to participate in the study, leaving a total of 375 respondents. 120 participants were recruited by the systematic random sampling method, and they were randomized into test and control groups of 60 participants each.

\section{Phase 1}

At first contact, phase 1 activities, which included pre-intervention and intervention activities, were undertaken.

\section{Pre-intervention activities}

Participants were made to sign a consent form to participate in the study. Each participant's socio-demographic characteristics were recorded. Both test and control groups received diabetes treatment, while participants in the test group were introduced to the study and were told to prepare to do Fasting Blood Glucose (FBG), 2 Hours Post Prandial (2HPP) and Glycosylated Hemoglobin $\left(\mathrm{HbA}_{1 \mathrm{c}}\right)$ tests in their next clinic visit two weeks later. The test group was encouraged to invite a close family member, preferably their spouse, as treatment partner to the clinic during their next visit. Each participant's mobile phone number was recorded for the purpose of follow up.

Two weeks later, each participant had his/her questionnaire completed. Their clinical parameters such as weight, height and calculation of body mass index (BMI) were measured into a section of the questionnaire. Blood samples for FPG, 2HPP and $\mathrm{HbA}_{1 \mathrm{c}}$ were taken.

\section{Diabetes self-care education}

In addition to the above, the test group had diabetes self-care training with the aid of the modified Diabetes Self-care Manual of the American Association of Diabetes Educator (AADE7) [10]. This was used to teach Diabetes Self-Management Education (DSME) to the test participants, along with their treatment partners. This pamphlet (AADE7) identified seven self-care behaviors, called the AADE7, which were: (i) Healthy eating: which recommended complex carbohydrates; (ii) Being active: which provided easy ideas for boosting physical activities; (iii) Self-monitoring of blood glucose: which recommended subjects to follow a schedule; (iv) Taking medication: as prescribed and having treatment support; (v) Problem solving: which required the subject to know how diabetes affects their body, to identify health problems and to learn how to solve them; (vi) Reducing risks: which taught subjects about potential complications and how to prevent them; and (vii) Healthy coping: which was aimed at coping with physical and emotional stress by building healthy relationships [15]. This was used to teach the respondents about diabetes self-care with the aid of pictures and diagrams contained therein. The activities were demonstrated to participants and their treatment partners, their possible constraints were discussed, and their questions were answered. They were given the AADE7 leaflets (appendix VII) as reminders and were encouraged to follow the diabetes self-care activities at home. The author also sent weekly text messages (SMS) to remind the participants about the activities.

Each member of the test group performed self-monitoring of blood glucose (SMBG) at home daily using a blood glucose-monitoring device (glucometer: Acucheck) and also kept a blood glucose/self-care diary, which they presented to the researcher at each clinic visit. The treatment partner aided the participant's understanding and subsequent compliance with the recommended self-care activities at home, reminded the participant about the self-care activities and helped monitor compliance with the activities.

Each member of the test group was scheduled for a four week follow up appointment. The diabetes self-care education program using AADE7 was repeated at each clinic visit to reinforce and encourage them to do the activities regularly. Each participant had an average of three clinic visits in the course of the research study. However, those who had other indications to attend more frequently were encouraged to do so.

The study was carried out in the five months between March and August 2014, and the recruitment of patients was done within the first two months. The recruitment was individualized such that each respondent was allowed to practice self-care activities for three months. At the end of the three months, participants were given appointment for one week to visit the clinic for phase 2, at which time the activities of phase 2 took place.

\section{Phase 2}

\section{Post intervention activities}

Each participant in the test group was seen for phase 2 at the end of his/her three months of self-care (phase 1) activities. The post-intervention (phase 2) activities included: (i) Administration of a second questionnaire - Diabetes self-care inventory manual, which was an expanded standardized self-reported questionnaire using the diabetes Self-Care Inventory Manual (SCI) developed by La Greca to assess the level of compliance to self-care activities during the study [16]. This was also used 
to assess the attitude toward diabetes self-care, diet adherence, exercise barrier scale and management practice scale (ii) Measurement of clinical parameters such as (a) Fasting Plasma Glucose (FPG), (b) two-hour postprandial glucose (2HPP) and (c) Glycosylated Hemoglobin $\left(\mathrm{HbA}_{1 \mathrm{c}}\right)$ to determine their level of glycemic control.

Each participant in the test group completed the diabetes self-care inventory questionnaire, and blood samples for FBG, $2 \mathrm{HPP}$ and $\mathrm{HbA}_{1 \mathrm{c}}$ were taken, with the results being recorded.

Plasma glucose level (fasting)

After an overnight fasting of approximately eight to ten hours, venous blood was collected from the participants using a $5 \mathrm{ml}$ hypodermic needle and syringe via the cubital fossa. The blood samples were sent to the hospital's laboratory for fasting plasma glucose determination using a Unico 2100 spectrophotometer (Canada).

Two-Hour Postprandial Blood Glucose (2HPP)

The 2HPP glucose test measures blood glucose exactly two hours after a meal. A blood sample was taken as above from the participants exactly two hours after their last meal [17]. The blood samples were sent to the hospital's laboratory, and the $2 \mathrm{HPP}$ of the samples was determined using a Unico 2100 spectrophotometer.

Glycosylated Hemoglobin (HBA $)$

$\mathrm{HbA}_{1 \mathrm{c}}$ was carried out using the $\mathrm{BIO}-\mathrm{RAD}$ in $2 \mathrm{it}^{\mathrm{TM}}$ (I) Analyzer and $A_{1 C}$ Test cartridges REF: 281-000 EX $[15,18]$. This was done in accordance with the established standard procedure and best practice. $\mathrm{HbA}_{1 c^{\prime} s}$ values less or equal to $6.5 \%$ were classified as good glycemic control, while those greater than $6.5 \%$ were considered as poor.

Self-Monitoring of Blood Glucose (SMBG) at Home

All participants were taught self-monitoring of plasma glucose using the glucometer. They were instructed to record their values in a provided logbook on at least two different days of the week. This record was brought to the clinic at the appropriate time for analysis. Home monitoring of plasma glucose was not required from the participants in the control group.

The following parameters were adopted for the purpose of this study.

Fasting Plasma Glucose (FPG) [19-21]

$<7.0 \mathrm{mmol} / \mathrm{l}-$ Good

$\geq 7.0 \mathrm{mmol} / \mathrm{l}-$ Poor

Two-Hour Postprandial (2HPP) [19, 21] $\leq 11.1 \mathrm{mmol} / \mathrm{l}-$ Good

$\mathrm{HbA}_{1 \mathrm{c}}[20-22]$

$>11.1 \mathrm{mmol} / \mathrm{l}-$ Poor

$<6.5 \%$ - Good

$\geq 6.5 \%$ - Poor

\section{Data analysis}

For the analysis of data, sections III, IV and V (III - Diabetes education and counselling received, IV - Understanding of diabetes and $\mathrm{V}$ - assessment of level of diabetes control) of the questionnaire were scored between 0 and 6 as appropriate, and the scores for each section were converted to percentages. A score less than 33\% was considered poor, between 34-65\% good, while scores greater than $66 \%$ were considered excellent. The data collected was analyzed using the Statistical Package for Social Sciences (SSPS) for Windows, software version 17.0. Two stage analysis was carried out, as well as analysis of the pre-intervention and post-intervention questionnaire. Means, medians, modes, standard deviations, proportions and percentages were determined. The test of significance was done using McNemar's Chi-square and Student's $t$-Test as appropriate. Chi-square was used to determine the statistical significance of observed differences in cross-tabulated variables. The Student's $t$-Test was used to compare the means when there were only two means to compare. A $p$-value equal or less than 0.05 was considered to be statistically significant. The results were illustrated using charts and tables.

\section{Ethical considerations}

Ethical approval was obtained from the Ethical Review and Research Committee of both the Federal Medical Center, Ido-Ekiti, and the Postgraduate Medical College of Nigeria. Informed verbal and written consent was obtained from willing participants. Patient confidentiality was maintained, and all data obtained was kept secured in the data store of the institution.

\section{Results}

\section{Socio-demographic findings}

Table 1 provides information regarding the 120 adult patients aged $30-80$ years. The mean age (SD) of the participants was 60.7 years \pm 7.5 . There were more female respondents ( 74 $(61.7 \%))$ than male $(46(38.3 \%))$, with a male to female ratio of $1: 1.6$. The majority of the respondents were Yorubas (90\%), Christians $(83.3 \%)$ and married $(80 \%)$ in a monogamous family type (75\%). Just over half (57.5\%) lived in rural areas of Ekiti.

The socio-demographic characteristics of the test group and the control group were similar. There was no statistically significant difference in the age $(p=0.374)$, gender $(p=0.060)$, marital status $(p=0.538)$, religion ( $p=0.142)$, ethnicity $(p=0.543)$,

\begin{tabular}{|c|c|c|c|c|c|c|}
\hline Variables & $\begin{array}{l}\text { Test group }(n=60) \\
n(\%)\end{array}$ & $\begin{array}{l}\text { Control group }(n=60) \\
n(\%)\end{array}$ & $\begin{array}{l}\text { Total }(n=120) \\
n(\%)\end{array}$ & $x^{2}$ & $D f$ & $p$ \\
\hline $\begin{array}{l}\text { Age in years } \\
\text { mean } \pm \text { SD* } \\
\text { (min-max) } \\
40-49 \\
50-59 \\
60-69 \\
\geq 70\end{array}$ & $\begin{array}{l}60.3 \pm 7.5 \\
(42-79) \\
4(6.7) \\
21(35.0) \\
29(48.3) \\
6(10.0)\end{array}$ & $\begin{array}{l}61.0 \pm 7.5 \\
(43-73) \\
6(10.0) \\
17(28.3) \\
25(41.7) \\
12(20.0)\end{array}$ & $\begin{array}{l}60.7 \pm 7.5 \\
(42-79) \\
10(8.3) \\
38(31.7) \\
54(45.0) \\
18(15.0)\end{array}$ & $\begin{array}{l}-0.509 \\
3.117\end{array}$ & $\begin{array}{l}118 \\
3\end{array}$ & $\begin{array}{l}0.612 \\
0.374\end{array}$ \\
\hline $\begin{array}{l}\text { Gender } \\
\text { male } \\
\text { female }\end{array}$ & $\begin{array}{l}28(46.7) \\
32(53.3)\end{array}$ & $\begin{array}{l}18(30.0) \\
42(70.0)\end{array}$ & $\begin{array}{l}46(38.3) \\
74(61.7)\end{array}$ & 3.525 & 1 & 0.060 \\
\hline $\begin{array}{l}\text { Marital Status } \\
\text { single } \\
\text { married } \\
\text { divorced } \\
\text { widowed }\end{array}$ & $\begin{array}{l}3(5.0) \\
50(83.3) \\
6(10.0) \\
1(1.7)\end{array}$ & $\begin{array}{l}6(10.0) \\
46(76.7) \\
5(8.3) \\
3(5.0) \\
\end{array}$ & $\begin{array}{l}9(7.5) \\
96(80.0) \\
11(9.2) \\
4(3.3)\end{array}$ & & & $0.538 * *$ \\
\hline $\begin{array}{l}\text { Religion } \\
\text { Christianity } \\
\text { Islam }\end{array}$ & $\begin{array}{l}53(88.3) \\
7(11.7)\end{array}$ & $\begin{array}{l}47(78.3) \\
13(21.7)\end{array}$ & $\begin{array}{l}100(83.3) \\
20(16.7)\end{array}$ & 2.160 & 1 & 0.142 \\
\hline
\end{tabular}




\begin{tabular}{|l|l|l|l|l|l|l|}
\hline Table 1. Socio-demographic characteristics of the study groups \\
\hline Variables & $\begin{array}{l}\text { Test group }(n=60) \\
n(\%)\end{array}$ & $\begin{array}{l}\text { Control group }(n=60) \\
n(\%)\end{array}$ & $\begin{array}{l}\text { Total }(n=120) \\
n(\%)\end{array}$ & $\chi^{2}$ & $D f$ \\
\hline $\begin{array}{l}\text { Ethnicity } \\
\text { Yoruba } \\
\text { Ibo }\end{array}$ & $\begin{array}{l}55(91.7) \\
5(8.3)\end{array}$ & $\begin{array}{l}53(88.3) \\
7(11.7)\end{array}$ & $\begin{array}{l}108(90.0) \\
12(10.0)\end{array}$ & 0.370 & 1 & 0.543 \\
\hline $\begin{array}{l}\text { Domicile } \\
\text { urban } \\
\text { rural }\end{array}$ & $23(38.3)$ & $\begin{array}{l}28(46.7) \\
32(53.3)\end{array}$ & $\begin{array}{l}51(42.5) \\
69(57.5)\end{array}$ & 0.853 & 1 & 0.356 \\
\hline
\end{tabular}

* Independent samples $t$-Test, ${ }^{* *}$ Fisher's exact test.

\begin{tabular}{|c|c|c|c|c|c|}
\hline Variables & Test group $(n=60)$ & Control group $(n=60)$ & $T$ & $D f$ & $p$ \\
\hline FPG (mmol/l) & $8.8 \pm 2.7$ & $9.8 \pm 3.0$ & -1.957 & 118 & 0.172 \\
\hline $2 \mathrm{HPP}(\mathrm{mmol} / \mathrm{l})$ & $13.1 \pm 3.8$ & $15.6 \pm 4.4$ & -3.398 & 118 & 0.140 \\
\hline $\mathrm{HbA}_{1 \mathrm{c}}(\%)$ & $6.7 \pm 1.1$ & $7.3 \pm 1.6$ & -2.330 & 118 & 0.272 \\
\hline Glycemic control & $n(\%)$ & $n(\%)$ & $x^{2}$ & $d f$ & $p$ \\
\hline \multicolumn{6}{|l|}{ FPG } \\
\hline Good & $17(28.3)$ & $11(18.3)$ & 1.677 & 1 & $0.195^{*}$ \\
\hline Poor & $43(71.7)$ & $49(81.7)$ & & & \\
\hline \multicolumn{6}{|l|}{$2 \mathrm{HPP}$} \\
\hline Good & $15(25.0)$ & 7 (11.7) & 3.562 & 1 & $0.059 *$ \\
\hline Poor & $45(75.0)$ & $53(88.3)$ & & & \\
\hline \multicolumn{6}{|l|}{$\mathrm{HbA}_{1 \mathrm{c}}$} \\
\hline Good & $32(53.3)$ & $26(43.3)$ & 1.201 & 1 & $0.273^{*}$ \\
\hline Poor & $28(46.7)$ & 34 (56.7) & & & \\
\hline
\end{tabular}

* Chi-square test.

domicile $(p=0.356)$, family type $(p=0.673)$ and household size $(p=0.853)$ of the respondents.

Figure 1 provides the duration of diabetes, where the majority of the participants $(70 \%$ of the intervention group and $58.3 \%$ of the control group) had diabetes for longer than five years. The duration of diabetes in both the intervention and control groups was similar. The difference was not statistically significant $(p>0.05)$.

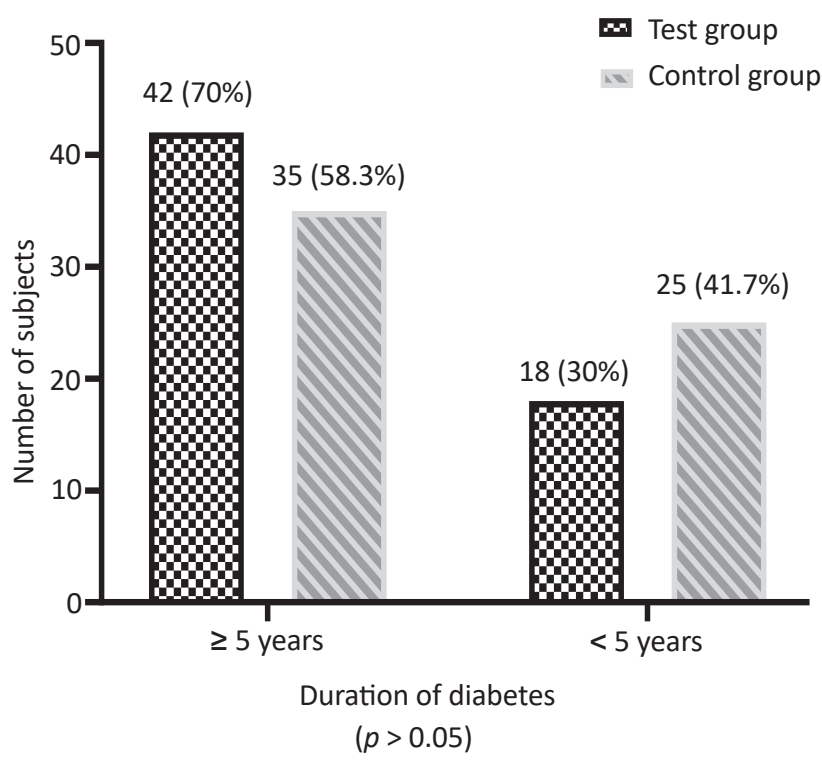

Figure 1. Clustered bar chart showing distribution of the participants by duration of diabetes since diagnosis
Pre-intervention blood glucose levels among the participants

The pre-intervention mean $\mathrm{HbA}_{1 \mathrm{c}}$ (\%) for the intervention and control groups was $6.7 \pm 1.1$ and $7.3 \pm 1.6$, respectively. However, there existed no significant difference between the intervention and control groups with regard to their pre-intervention glycemic control ( $p=0.272$ ) (Table 2 ).

\section{Pre-intervention level of diabetes knowledge among the participants}

Only about $32(26.7 \%)$ of the participants had ever had any diabetes education before the intervention. The table showed no statistically significant difference $(p=0.409)$ between the intervention and control groups based on their diabetes education and understanding of diabetes care (Table 3).

\begin{tabular}{|c|c|c|c|c|c|c|}
\hline \multicolumn{2}{|c|}{$\begin{array}{l}\text { Test group } \\
n=60(\%)\end{array}$} & $\begin{array}{l}\text { Control } \\
\text { group } \\
n=60(\%)\end{array}$ & $\begin{array}{l}\text { Total } \\
(n=120)\end{array}$ & $x^{2}$ & $D f$ & $p$ \\
\hline \multicolumn{2}{|c|}{$\begin{array}{l}\text { Ever had diabetes } \\
\text { education }\end{array}$} & & & \multirow{3}{*}{0.682} & \multirow{3}{*}{1} & \multirow{3}{*}{0.409} \\
\hline Yes & $18(30.0)$ & $14(23.3)$ & $32(26.7)$ & & & \\
\hline No & $42(70.0)$ & 46 (76.7) & $88(73.3)$ & & & \\
\hline \multicolumn{2}{|c|}{$\begin{array}{l}\text { Understanding of } \\
\text { DM care process }\end{array}$} & & & \multirow{3}{*}{0.363} & \multirow{3}{*}{1} & \multirow{3}{*}{0.547} \\
\hline Good & $16(26.7)$ & 19 (31.7) & $35(29.2)$ & & & \\
\hline Poor & $44(73.3)$ & $41(68.3)$ & 85 (70.8) & & & \\
\hline
\end{tabular}




\section{Pre-intervention diabetes self-care practices among the participants}

In all pre-intervention diabetes self-care practices, there existed no significant difference between the test and the control groups in all variables that assessed the pattern of diabetes self-care, except in footcare $(p=0.007)$ (Table 4$)$.

\section{Post-intervention blood glucose levels among the participants}

The results for post-intervention blood glucose levels showed that there was significant difference (FPG $(p=0.025)$; 2 HPP ( $p=$ $0.025) ; \mathrm{HbA}_{1 c}(p<0.001)$ between the test and control group in all parameters of glycemic control (Table 5).

\section{Effects of intervention on glycemic control in the test group}

The effect of intervention between the pre- and post-intervention groups demonstrated a significant difference in FPG $(p=0.010)$ and $\operatorname{HBA}_{1 c}(p=0.001)$. However, there existed no significant difference in 2HPP $(p=0.060)$ (Table 6).

\section{Initial and final differences in blood glucose levels among control respondents}

There existed no significant difference in all the parameters of glycemic variables (FPG $(p=1.355), 2 \mathrm{HPP}(p=0.369)$ and $\left.\operatorname{HBA}_{1 c}(p=0.804)\right)$ in the initial and final glycemic values in the control group (Table 7).

\begin{tabular}{|c|c|c|c|c|c|c|}
\hline Variables & $\begin{array}{l}\text { Study group } \\
(n=60) \\
n(\%)\end{array}$ & $\begin{array}{l}\text { Control group } \\
(n=60) \\
n(\%)\end{array}$ & $\begin{array}{l}\text { Total } \\
(n=120) \\
n(\%)\end{array}$ & $x^{2}$ & $D f$ & $p$ \\
\hline $\begin{array}{l}\text { Tests blood sugar } \\
\text { yes } \\
\text { no }\end{array}$ & $\begin{array}{l}38(63.3) \\
22(36.7)\end{array}$ & $\begin{array}{l}33(55.0) \\
27(45.0)\end{array}$ & $\begin{array}{l}71(59.2) \\
49(40.8)\end{array}$ & 0.862 & 1 & 0.355 \\
\hline $\begin{array}{l}\text { Keeps records of test results }(n=71) \\
\text { yes } \\
\text { no }\end{array}$ & $\begin{array}{l}12(31.6) \\
26(68.4) \\
\end{array}$ & $\begin{array}{l}5(15.2) \\
28(84.8)\end{array}$ & $\begin{array}{l}17 \text { (23.9) } \\
54 \text { (76.1) } \\
\end{array}$ & 2.617 & 1 & 0.106 \\
\hline $\begin{array}{l}\text { Takes medication as prescribed } \\
\text { yes } \\
\text { no }\end{array}$ & $\begin{array}{l}34(56.7) \\
26(43.3)\end{array}$ & $\begin{array}{l}29(48.3) \\
31(51.7)\end{array}$ & $\begin{array}{l}63(52.5) \\
57(47.5)\end{array}$ & 0.835 & 1 & 0.361 \\
\hline $\begin{array}{l}\text { Risk reduction (foot care) } \\
\text { yes } \\
\text { no }\end{array}$ & $\begin{array}{l}27(45.0) \\
33(55.0)\end{array}$ & $\begin{array}{l}13(21.7) \\
47(78.3)\end{array}$ & $\begin{array}{l}40(33.3) \\
80(66.7)\end{array}$ & 7.350 & 1 & 0.007 \\
\hline $\begin{array}{l}\text { Exercise program } \\
\text { yes } \\
\text { no }\end{array}$ & $\begin{array}{l}24(40.0) \\
36(60.0)\end{array}$ & $\begin{array}{l}21(35.0) \\
39(65.0)\end{array}$ & $\begin{array}{l}45(37.5) \\
75(62.5)\end{array}$ & 0.320 & 1 & 0.571 \\
\hline $\begin{array}{l}\text { Meal plans } \\
\text { yes } \\
\text { no }\end{array}$ & $\begin{array}{l}5(8.3) \\
55(91.7)\end{array}$ & $\begin{array}{l}7(11.7) \\
53(88.3)\end{array}$ & $\begin{array}{l}12(10.0) \\
108(90.0)\end{array}$ & 0.370 & 1 & 0.543 \\
\hline $\begin{array}{l}\text { Problem solving skill } \\
\text { yes } \\
\text { no }\end{array}$ & $\begin{array}{l}19(31.7) \\
41(68.3)\end{array}$ & $\begin{array}{l}22(36.7) \\
38(63.3)\end{array}$ & $\begin{array}{l}41(34.2) \\
79(65.8)\end{array}$ & 0.333 & 1 & 0.564 \\
\hline $\begin{array}{l}\text { Healthy coping skill } \\
\text { yes } \\
\text { no }\end{array}$ & $\begin{array}{l}5(8.3) \\
55(91.7)\end{array}$ & $\begin{array}{l}9(15.0) \\
51(85.0)\end{array}$ & $\begin{array}{l}14(11.7) \\
106(88.3)\end{array}$ & 1.294 & 1 & 0.255 \\
\hline
\end{tabular}

\begin{tabular}{|c|c|c|c|c|c|}
\hline & Test group $(n=60)$ & Control group $(n=60)$ & $T$ & $D f$ & $p$ \\
\hline FPG (mmol/l) & $6.3 \pm 1.4$ & $9.1 \pm 2.6$ & -7.163 & 118 & 0.021 \\
\hline $2 \mathrm{HPP}(\mathrm{mmol} / \mathrm{l})$ & $11.4 \pm 1.6$ & $14.7 \pm 3.5$ & -6.763 & 118 & 0.014 \\
\hline $\mathrm{HbA}_{1 \mathrm{c}}(\%)$ & $5.6 \pm 1.0$ & $6.8 \pm 1.3$ & -5.445 & 118 & $<0.001$ \\
\hline Glycemic control & $n(\%)$ & $n(\%)$ & $x^{2}$ & $d f$ & $p$ \\
\hline $\begin{array}{l}\text { FPG } \\
\text { good } \\
\text { poor }\end{array}$ & $\begin{array}{l}31(51.7) \\
29(48.3) \\
\end{array}$ & \begin{tabular}{|l|}
$18(30.0)$ \\
$42(70.0)$ \\
\end{tabular} & 9.766 & 1 & $0.025^{*}$ \\
\hline $\begin{array}{c}2 \mathrm{HPP} \\
\text { good } \\
\text { poor }\end{array}$ & $\begin{array}{l}28(46.7) \\
32(53.3) \\
\end{array}$ & \begin{tabular}{|l}
$16(26.7)$ \\
$44(73.3)$ \\
\end{tabular} & 5.167 & 1 & $0.025^{*}$ \\
\hline $\begin{array}{c}\mathrm{HbA}_{1 \mathrm{c}} \\
\text { good } \\
\text { poor }\end{array}$ & $\begin{array}{l}51(85.0) \\
9(15.0)\end{array}$ & $\begin{array}{l}28(46.7) \\
32(53.3)\end{array}$ & 19.599 & 1 & $<0.001 *$ \\
\hline
\end{tabular}

\footnotetext{
* Chi-square test.
} 


\begin{tabular}{|c|c|c|c|c|c|}
\hline Variables & Test pre-intervention & Test post-intervention & $T^{*}$ & $D f$ & $p$ \\
\hline FPG (mmol/l) & $8.8 \pm 2.7$ & $6.3 \pm 1.4$ & 6.811 & 59 & 0.013 \\
\hline $2 \mathrm{HPP}(\mathrm{mmol} / \mathrm{l})$ & $13.1 \pm 3.8$ & $11.4 \pm 1.6$ & 3.645 & 59 & 0.041 \\
\hline $\mathrm{HbA}_{1 \mathrm{c}}(\%)$ & $6.7 \pm 1.1$ & $5.6 \pm 1.0$ & 6.495 & 59 & 0.002 \\
\hline Glycemic control & $n(\%)$ & $n(\%)$ & $x^{2}$ & $d f$ & $p$ \\
\hline $\begin{array}{l}\text { FPG } \\
\text { good } \\
\text { poor }\end{array}$ & $\begin{array}{l}17(28.3) \\
43(71.7) \\
\end{array}$ & $\begin{array}{l}31(51.7) \\
29(48.3) \\
\end{array}$ & 6.806 & 1 & $0.010 * *$ \\
\hline $\begin{array}{l}2 \mathrm{HPP} \\
\text { good } \\
\text { poor }\end{array}$ & $\begin{array}{l}18(30.0) \\
42(70.0)\end{array}$ & $\begin{array}{l}28(46.7) \\
32(53.3)\end{array}$ & 3.525 & 1 & $0.060 * *$ \\
\hline $\begin{array}{r}\mathrm{HbA}_{1 \mathrm{c}} \\
\text { good } \\
\text { poor }\end{array}$ & $\begin{array}{l}32(53.3) \\
28(46.7)\end{array}$ & $\begin{array}{l}51(85.0) \\
9(15.0)\end{array}$ & 14.106 & 1 & $<0.001 * *$ \\
\hline
\end{tabular}

* Paired samples $t$-Test, ${ }^{* *}$ Chi-square.

\begin{tabular}{|c|c|c|c|c|c|}
\hline \multirow[t]{2}{*}{ Variables } & \multicolumn{2}{|c|}{ Control group } & \multirow[t]{2}{*}{$T^{*}$} & \multirow[t]{2}{*}{$D f$} & \multirow[t]{2}{*}{$p$} \\
\hline & Initial & Final & & & \\
\hline FPG $(\mathrm{mmol} / \mathrm{l})$ & $9.8 \pm 3.0$ & $9.1 \pm 2.6$ & 1.953 & 59 & 0.056 \\
\hline $2 \mathrm{HPP}(\mathrm{mmol} / \mathrm{l})$ & $15.6 \pm 4.4$ & $14.7 \pm 3.5$ & 1.580 & 59 & 0.120 \\
\hline $\mathrm{HbA}_{1 \mathrm{c}}(\%)$ & $7.3 \pm 1.6$ & $6.8 \pm 1.3$ & 2.560 & 59 & 0.013 \\
\hline Glycemic control & $n(\%)$ & $n(\%)$ & $\chi^{2}$ & $d f$ & $p$ \\
\hline $\begin{array}{l}\text { FPG } \\
\text { good } \\
\text { poor }\end{array}$ & $\begin{array}{l}11 \text { (18.3) } \\
49(81.7)\end{array}$ & $\begin{array}{l}18(30.0) \\
42(70.0)\end{array}$ & 2.228 & 1 & $1.355^{* *}$ \\
\hline $\begin{array}{l}2 \mathrm{HPP} \\
\text { good } \\
\text { poor }\end{array}$ & $\begin{array}{l}7(11.7) \\
53(88.3)\end{array}$ & $\begin{array}{l}16(26.7) \\
44\end{array}$ & 4.357 & & $0.369 * *$ \\
\hline $\begin{array}{r}\mathrm{HbA}_{1 \mathrm{c}} \\
\text { good } \\
\text { poor }\end{array}$ & $\begin{array}{l}26(43.3) \\
34(56.7)\end{array}$ & $\begin{array}{l}28(46.7) \\
32(53.3)\end{array}$ & & & $0.804^{* *}$ \\
\hline
\end{tabular}

* Paired samples $t$-Test, ${ }^{* *}$ Chi-square.

\begin{tabular}{|l|l|l|l|l|l|}
\hline Table 8. Differences in post-intervention blood glucose level by level of diabetes self-care \\
\hline \multirow{2}{*}{ Post-intervention blood sugar levels } & Diabetes self-care & $T$ & Df & $p$ \\
\cline { 2 - 6 } & Good & Poor & & & \\
\hline FPG (mmol/l) & $6.2 \pm 1.2$ & $7.1 \pm 1.4$ & -2.309 & 58 & 0.025 \\
\hline $2 \mathrm{HPP}(\mathrm{mmol} / \mathrm{l})$ & $11.0 \pm 1.1$ & $11.7 \pm 1.0$ & -2.068 & 58 & 0.043 \\
\hline $\mathrm{HbA}_{1 \mathrm{c}}(\%)$ & $5.4 \pm 1.0$ & $6.2 \pm 1.4$ & -2.515 & 58 & 0.015 \\
\hline
\end{tabular}

\begin{tabular}{|c|c|c|c|c|c|c|}
\hline \multirow[b]{3}{*}{ Diabetic self-care } & \multicolumn{3}{|c|}{ Post-intervention Glycemic Control } & \multirow[t]{3}{*}{$x^{2}$} & \multirow[t]{3}{*}{$D f$} & \multirow[t]{3}{*}{$p$} \\
\hline & \multicolumn{3}{|l|}{ FPG } & & & \\
\hline & Good $n(\%)$ & & Poor $n(\%)$ & & & \\
\hline Good & $29(60.4)$ & & $19(39.6)$ & & & $0.010^{*}$ \\
\hline Poor & $2(16.7)$ & & $10(83.3)$ & & & \\
\hline Total & 31 (61.7) & 2HPP & $29(21.7)$ & & & \\
\hline Diabetic self-care & Good $n(\%)$ & & Poor $n(\%)$ & & & \\
\hline Good & $26(55.3)$ & & 21 (44.7) & 6.525 & 1 & 0.011 \\
\hline Poor & $2(15.4)$ & & 11 (84.6) & & & \\
\hline Total & $28(46.7)$ & $\mathrm{HbA}_{1 \mathrm{c}}$ & 32 (53.3) & & & \\
\hline Diabetic self-care & Good $n(\%)$ & & Poor $n(\%)$ & & & \\
\hline Good & $43(91.5)$ & & $4(8.5)$ & & & $0.018^{*}$ \\
\hline Poor & $8(61.5)$ & & 5 (38.5) & & & \\
\hline Total & $51(85.0)$ & & $9(15.0)$ & & & \\
\hline
\end{tabular}

* Fisher's exact test. 
Differences in post-intervention blood glucose level by level of diabetes self-care

This table shows a significant relationship between blood glucose levels and diabetes self-care in the test group (Table 8).

\section{Relationship between diabetes self-care and glyce- mic control among the test group}

This table shows a significant relationship (FPG $(p=0.025)$; $\left.2 \mathrm{HPP}(p=0.043) ; \operatorname{HBA}_{1 c}(p=0.015)\right)$ between blood glucose levels and diabetes self-care in the test group (Table 9).

\section{Effect of intervention on glycemic control $\left(\mathrm{HbA}_{1 \mathrm{c}}\right)$ among the test group}

After the intervention, the number of respondents with good glycemic control increased from 32 (53.3\%) to 51 (85\%), while the number with poor glycemic control decreased from $28(46.7 \%)$ to $9(15 \%)$. This change was statistically significant $(p<0.001)$ and showed that there was a significant relationship between glycemic control and diabetes self-care practice (Figure 2).

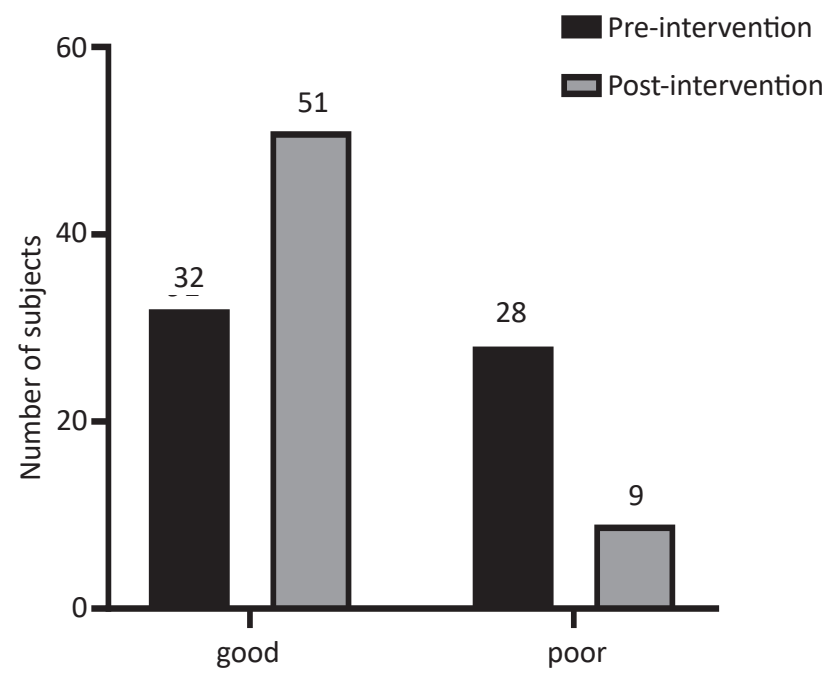

Glycemic control

Figure 2. Effect of intervention on glycemic control $\left(\mathrm{HbA}_{1 \mathrm{c}}\right)$ among the test group $(p<0.001)$

\section{Discussion}

The socio-demographic profile of the participants in this study showed that the majority were in the age group of 60 to 69 years with a mean age (SD) of 60.7 years $( \pm 7.5)$. This may be due to Ekiti towns being mostly rural and semi-urban settlements with a sizeable number of middle age and elderly citizens. The mean age of this study was higher than the mean age of 58.3 years $( \pm 1.2)$ and 53.4 years $( \pm 10.9)$ reported in similar studies in Benin city and San Francisco, respectively [23, 24]. A good number (57.7\%) of the participants in this study lived in rural areas, Ido-Ekiti and its environs, compared to Benin and San Francisco, which were urban and industrialized cities. This lends credibility to the submission that urbanization is a recognized factor in the increasing prevalence of diabetes [23, 25].

The age distribution of the participants in this study demonstrated that the number of people living with diabetes mellitus increases with age, but it is most prevalent among those aged 50-69. This was consistent with report of studies in lle-Ife and Benin $[25,26]$, and it supported the assertion that diabetes was not just a disease of the elderly, but it is also common among those of middle age [27]. In the present study, there were more female participants than males. The percentage of females was $61.7 \%$, greater than the $38.3 \%$ of males, and the ratio was $1.6: 1$. This higher percentage of females may be due to more health-seeking behaviors in women than men, and it is similar to what was reported in Ife, Nnewi and Benin [23, 25, 27]. One-third $(34.2 \%)$ of the participants in the current study attained tertiary level education. This was similar to findings from an earlier study in the same center [24], which reported that education and income are major socio-economic determinants of health and that poor educational attainment and low socio-economic status were significantly associated with lower knowledge and practice of diabetes self-care [28].

This study showed that glycemic control was poor among the respondents before the intervention. The pre-intervention mean FPG $(\mathrm{mmol} / \mathrm{l})$ of the test group was $8.8 \pm 2.7$, while that of the control group was $9.8 \pm 3.0$, and $\mathrm{HbA}_{1 \mathrm{c}}(\%)$ was $6.7 \pm 1.1$ among the test group and $7.3 \pm 1.6$ among the control group. The percentage of those with poor glycemic control was high in both groups in the pre-intervention period. This is similar to that reported in a multicenter study which examined the profile of Nigerians with diabetes mellitus and another study which looked at glycemic control in patients with type 2 diabetes mellitus in Benin and Ilorin $[23,29]$. Only $53.3 \%$ of the test and $43.3 \%$ of the control groups met the $6.5 \% \mathrm{HbA}_{1 c}$ glycemic control value recommended by the World Health Organization [21]. The pre-intervention mean $\mathrm{FBG}$ and $\mathrm{HbA}_{1 c}$ is an indication that achieving good glycemic control still appear to be a challenge among diabetics in Nigeria.

Post-intervention glycemic control in the test group with mean FPG $(\mathrm{mmol} / \mathrm{l})$ values of $6.3 \pm 1.4$, mean $2 \mathrm{HPP}(\mathrm{mmol} / \mathrm{l})$ $11.4 \pm 1.6$ and mean $\mathrm{HbA}_{1 \mathrm{c}}$ (\%) $5.6 \pm 1.0$, compared to that of the final blood glucose levels in the control group with mean FPG, $2 \mathrm{HPP}$ and $\mathrm{HbA}_{1 \mathrm{c}}$ values of $9.1 \pm 2.6,14.7 \pm 3.5$ and $6.8 \pm 1.3 \%$, respectively, was statistically significant (FPG with $p=0.021$; $2 \mathrm{HPP}$ with $p=0.041 ; \mathrm{HbA}_{1 \mathrm{c}}$ with $p<0.001$ ). This showed that diabetes self-care practice had a significant effect on the blood glucose level of the test group. The proportion of those who achieved good glycemic control among the test group increased from $53.3 \%$ to $85.0 \%$, while that of the control group marginally decreased from $46.7 \%$ to $43.3 \%$. This result among the control group could be due to the fact that they maintained their usual traditional way of diabetes care, having neither undergone diabetes self-care education nor practiced diabetes self-care. The result is comparable to findings in a study in which diabetes mellitus was monitored with $\mathrm{HbA}_{1 \mathrm{c}}$ in Abakaliki, where a mean $\mathrm{HbA}_{1 \mathrm{c}}$ of $6.59 \pm 1.02$ was reported among the diabetes population who had good glycemic control [30]. This finding is consistent with the report of Olorunsola, who, in a similar study, found that an increase in self-care practice resulted in a significant improvement in glycemic control [31].

This study showed low levels of diabetes knowledge and diabetes self-care practices among the participants in the pre-intervention period; however, there was a marked improvement in the level of knowledge and practice of diabetes self-care among the test group compared to the control group post-intervention. $38(63.3 \%)$ of the test group now had diabetes education compared to 20 (33.3\%) of the control ( $p=0.001) .35$ $(58.3 \%)$ of the test group had a good understanding of the diabetes care process compared to $21(35.0 \%)$ among the control group $(p=0.010)$. The intervention provided in this study was diabetes self-care, which was taught to the participants through Diabetes Self-Management Education (DSME) with the aid of AADE7 and targeted a change in the health behaviors of the participants through daily and regular practice of diabetes self-care activities. This was consistent with findings in North America, where a significant relationship between diabetes knowledge and the practice of diabetes self-care was reported [32]. Essien et al. demonstrated that guideline-based DSME intervention is effective in improving glycemic control and preventing diabe- 
tes complications among patients with type 2 diabetes mellitus [33]. However, a review by Stephani et al. revealed that self-management in Sub-Sahara Africa is poor and could pose a serious threat to the healthcare system [34].

When comparing the initial and final glucose levels among the test and control groups in the pre- and post-intervention period, a significant reduction in the glucose level from the preto post-intervention by $8.8 \pm 2.7$ to $6.3 \pm 1.4 \mathrm{mmol} / \mathrm{l}(p=0.013)$ for mean FPG, from $13.1 \pm 3.8$ to $11.4 \pm 1.6 \mathrm{mmol} / \mathrm{I}(p=0.041)$ for mean $2 \mathrm{HPP}$ and from $6.7 \pm 1.1 \%$ to $5.6 \pm 1.0 \%(p=0.002)$ for mean $\mathrm{HbA}_{1 \mathrm{c}}$ translated to significantly improved glycemic control in the test group. This was in comparison to the initial $9.8 \pm 3.0$ to final $9.1 \pm 2.6 \mathrm{mmol} / \mathrm{l}(p=0.056)$ for $\mathrm{FPG}$, from 15.6 \pm 4.4 to $14.7 \pm 3.5 \mathrm{mmol} / \mathrm{l}(p=0.120)$ for mean $2 \mathrm{HPP}$ and from $7.3 \pm 1.6$ to final $6.8 \pm 1.3 \%$ for mean $\mathrm{HbA}_{1 \mathrm{c}}$ among the control group, which represented a marginal change. This was further demonstrated by a significant difference in the proportion of those who achieved good FPG from $28.3 \%$ pre-intervention to $51.7 \%$ post-intervention ( $p=0.009$ ), 2 HPP from $30 \%$ to $46.7 \%$ ( $p=0.060$ ) and $\mathrm{HbA}_{1 \mathrm{c}}$ from $53.3 \%$ to $85 \%$ in the test group, while that of the control was FPG $18.3 \%$ to $30 \%(p=1.355)$, $2 \mathrm{HPP} 11.7 \%$ to $26.7 \%(p=0.369)$ and $\mathrm{HbA}_{1 \mathrm{c}} 43.3 \%$ to $53.3 \%$ ( $p=0.804$ ). Moreover, in this study, $85 \%$ of the test group had an $\mathrm{HbA}_{1 \mathrm{c}}$ value less than the $6.5 \%$ recommended by the World Health Organization $(p<0.01)$ compared to $28.3 \%$ in the preintervention period, and $46.7 \%(p=0.804)$ of the control had an $\mathrm{HbA}_{1 \mathrm{c}}$ value less than $6.5 \%$ compared to their initial $43.3 \%$. This showed a significant improvement in the glycemic control of the test group compared to the control group, which may be connected with the changes in lifestyle brought about by the practice of diabetes self-care among the test group.

In this study, among the test group, clear differences could be seen between those who had good and poor practices of diabetes self-care, with a mean FPG $(\mathrm{mmol} / \mathrm{l})$ of $6.2 \pm 1.2$ for good practice and $7.1 \pm 1.4(p=0.025)$ for poor practice of diabetes self-care, a mean $2 \mathrm{HPP}(\mathrm{mmol} / \mathrm{l})$ of $11.0 \pm 1.1$ for good practice compared to $11.7 \pm 1.0(p=0.043)$ for poor practice and a mean $\mathrm{HbA}_{1 \mathrm{c}}(\%)$ of $5.4 \pm 1.0$ compared to $6.2 \pm 1.4(p=0.015)$ for those with poor diabetes self-care practices. This is further corroborated by the proportion of those who achieved good practice of diabetes self-care to those who did not among test group. Almost two-thirds $(29 ; 60.4 \%)$ of those who had good diabetes self-care achieved good control in their fasting plasma glucose compared to just over one-third $(19 ; 39.6 \%)$ those who do not, and $26(55.3 \%)$ of those with good self-care practice had good control of their 2HPP compared to 21 (44.7\%) of those who did not, while $43(91.5 \%)$ of those who had good practice of diabetes self-care achieved good control in their $\mathrm{HbA}_{1 \mathrm{c}}$ values. These results showed positive and significant relationships between good practice of diabetes self-care and glycemic control [35,
36]. This was further supported by the result of a similar study conducted by Ugwu et al., who found that diabetes care knowledge and practice were poor even among Primary Health Care Physicians in Southwest Nigeria [37]. A study done by Tewahido et al. in Addis Ababa also concluded that self-care practices for effective management of diabetes is not adequately emphasized in diabetes care centers, and most patients lack sufficient knowledge for proper self-care [38].

In the current study, it is evident that among the participants with good diabetes self-care, 43 (91.5\%) had good glycemic $\left(\mathrm{HbA}_{11}\right)$ control, while $8.5 \%$ had poor control. This showed a statistically significant relationship between glycemic control and diabetes self-care (FBG; $p=0.01$ and $\mathrm{HbA}_{1 c^{\prime}} ;<0.018$ ). The improvement could have been due to improved diabetes self-care following intervention. The level of compliance among respondents in this study could be attributable to the close follow up by weekly phone calls from the author to ensure respondents' compliance to the self-care regimen, as well as the involvement of treatment partners in the form of a respondent's spouse or close relative who daily reminded the test participants of their responsibility towards self-care practice. This was at opposition with the reported $30 \%$ compliance to self-care in a similar study in India [39].

Cumulatively, those with good diabetes self-care practice were five times more likely to achieve good glycemic control. However, simultaneous practice of the components of diabetes self-care exerted a more significant effect on the outcome of glycemic control. Hence, the findings in this study have demonstrated that the knowledge and subsequent practice of diabetes self-care positively impacted the health status of respondents by improving their glycemic control.

\section{Conclusions}

The diabetes self-care education given to the test group and the enablement of the same group to transform the knowledge gained into diabetes self-care activities had a positive impact on the glycemic control of the respondents. We therefore conclude that the practice of diabetes self-care is an important component of diabetes management to achieve the desired glycemic control and prevent dreaded diabetes complications. Therefore, it is important that healthcare providers actively involve the patient in developing a self-care regimen with the best possible combination which should sound realistic to the patient so that it can be followed easily. This will contribute greatly to achieving good glycemic control and thereby eliminate acute complications and reduce the occurrence of chronic complications of the disease in such individuals, which will have a corresponding impact on health facilities and resources.

Source of funding: This work was funded from the authors' own resources.

Conflicts of interest: The authors declare no conflicts of interest.

\section{References}

1. American Diabetes Association. Diagnosis and classification of diabetes mellitus. Diabetes Care 2011; 34(1): S62-S69.

2. Hall V, Thomsen RW, Henriksen O, et al. Diabetes in Sub Saharan Africa 1999-2011: epidemiology and public health implications. A systematic review. BMC Public Health 2011; 11: 564-572.

3. Heneghan C, Blacklock C, Perera R. Evidence for non-communicable diseases: analysis of Cochrane reviews and randomized trials by World Bank classification. BMJ 2013; 3: 1-10.

4. Probst-Hensch N, Tanner M, Kessler $\mathrm{C}$, et al. Prevention - a cost-effective way to fight the non-communicable disease epidemic. An academic perspective of the United Nations High-Level NCD Meeting. Swiss Med Wkly 2011; 141: 1-8.

5. Nur K, Bariyyoh MH, Bujang MA, et al. Self-care activities among diabetic patients and factors affecting glycaemic control in Primary Health Care, Malaysia. Journal of Diabetes and Clinical Practices 2018; 1 : 1.

6. Collins MM, Bradley CP, O'Sullivan T, et al. Self-care coping strategies in people with diabetes: a qualitative exploratory study. BMC Endocrine Disorders 2009; 9: 6-8.

7. Daly JM, Hartz AJ, Yinghui XU, et al. Assessment of attitudes, behaviors, and outcomes of patients with type 2 diabetes. J Am Board Fam Med 2009; 22(3): 280-290.

8. Heisler M. Overview of peer support models to improve diabetes self-management and clinical outcomes. Diabetes Spectrum 2007; 20(4): 214-221. 
9. Epstein RM, Fiscella K, Lesser CS, et al. Why the nation needs a policy push on patient-centered health care. Health Affairs 2010; 29(8): 1489-1495.

10. American Association of Diabetes Educators: AADE7 Self-Care Behaviors. Diabetes Educ 2008; 34: 445-449.

11. American Association of Diabetes Care. Clinical practice recommendations. Diabetes Care 2009; 32: 541-546.

12. Adibe MO, Aguwa CN, Ukwe CV. The construct validity of an instrument for measuring type 2 diabetes self-care knowledge in Nigeria. Trop J Pharm Res 2011; 10(5): 619-629.

13. Inzucchi SE, Bergenstal RM, Buse JB, et al. Management of hyperglycemia in type 2 diabetes: a patient-centered approach position statement of the American Diabetes Association (ADA) and the European Association for the Study of Diabetes (EASD). Diabetes Care 2012; 35(6): 1364-1379.

14. World health Organization. Use of Glycated Haemoglobin $\left(\mathrm{HbA}_{1 \mathrm{c}}\right)$ in the Diagnosis of Diabetes Mellitus: Abbreviated Report of a WHO consultation. WHO 2011: 1-25. Available from URL: http://who.int/.../report-hba1c-2011.pdf.

15. Raithatha SJ, Shankar SU, Dinesh K. Self-care practices among diabetic patients in Anand District of Gujarat. ISRN Fam Med 2014; (2014)743791: 1-6.

16. Katie W, Heather AB, Annette M. La Greca. Measuring diabetes self-care: a psychometric analysis of the Self-Care Inventory-revised with adults. Diabetes Care 2005; 28(6): 1346-1352.

17. Chinenye S, Young E. Diabetes care in Nigeria. TNHJ 2011; 11(4): 101-106.

18. Yeo CP, Tan $\mathrm{CH}$, Jacob E. Haemoglobin $\mathrm{A}_{1 \mathrm{c}}$ : evaluation of a new $\mathrm{HbA}_{1}$ point-ofcare analyzer Bio-Rad In2it in comparison with the DCA 2000 and central laboratory analyzers. Ann Clin Biohem 2009; 46: 373-376.

19. Kitabchi AE, Umpierrez GE, Miles JM, et al. Hyperglycemic crises in adult patients with diabetes. Diabetes Care 2009; 32: 1335-1343.

20. Zhang X, Gregg EW, Williamson DF. A ${ }_{11}$ level and future risk of diabetes: a systematic review. Diabetes Care 2010; 33: $1665-1673$.

21. Rodrigues FFL, Zanetti ML, Santos MA, et al. Knowledge and attitude: important components in diabetes education. Rev Lat-Am Enferm 2009; 17(4): 468-473.

22. International Expert Committee. International Expert Committee report on the role of the $\mathrm{HbA}_{1 \mathrm{c}}$ assay in the diagnosis of diabetes. Diabetes Care 2009; 32: 1327-1334.

23. Adewolu OF. Glycaemic control in patients with type 2 diabetes mellitus in a secondary and tertiary health center in Oredo and Egor Local Government Areas in Benin City, South-South Nigeria. IJTDH 2014; 4(6): 621-633.

24. Wild S, Roglic G, Green A, et al. Global prevalence of diabetes estimates for the year 2000 and projections for 2030. Diabetes Care 2006; 27: 1047-1053.

25. Oyegbade OO, Abioye Kuteyi EA, Kolawole BA, et al. Screening for diabetes mellitus in a Nigerian family medicine population. SA Fam Pract 2007; 49(8): 15-22.

26. Unadike BC, Eregie A, Ohwovoriole AE. Glycaemic control amongst persons with diabetes mellitus in Benin City. Niger Med J 2010; 51: 164-166.

27. Pascal IG, Ofoedu JN, Uchenna NP, et al. Blood glucose control and medication adherence among adult type 2 diabetic Nigerians attending a primary care clinic in under-resourced environment of eastern Nigeria. North Am J Med Sci 2012; 4: 310-315.

28. Desalu OO, Salawu FK, Jimoh AK, et al. Diabetic foot care: self-reported knowledge and Practice among patients attending three tertiary Hospital in Nigeria. GMJ 2011; 45(2): 60-65.

29. Adebisi SA, Oghagbon EK, Akande TM, et al. Glycated haemoglobin and glycaemic control of diabetes in Ilorin. Niger J Clin Pract 2009; 12(1): 87-91.

30. Adebayo KJ. Monitoring diabetes mellitus with $\mathrm{HBA}_{1 \mathrm{c}}$ : the Abakaliki, Nigeria Experience. JHMN 2015; 12: $2422-8419$.

31. Modupe OA. Self-care practice and glycaemic control among type 2 diabetes mellitus patients in a secondary health care in Southwest, Nigeria. Arch Gen Inter Med 2017; 1(3): 48.

32. Osborn CY, Bains SS, Egede LE. Health literacy, diabetes self-care and glycemic control in adults with type 2 diabetes. Diabetes Technol Ther 2010; 12(11): 913-919.

33. Essien $\mathrm{O}$, Out A, Umoh V, et al. Intensive patient education improves glycaemic control in diabetes mellitus compared to conventional education: a randomized control trial in Nigeria tertiary care Hospital. PLoS ONE 2017; 12(1): e016885.

34. Stephani V, Opoku D, Beran D. Self-management of diabetes in Sub-Sahara Africa: a systematic review. BMC Public Health 2018; 18: 1148.

35. Leyva B, Zagarins SE, Allen NA, et al. The relative impact of diabetes distress versus depression on glycaemic control in Hispanic patients following a diabetes self-management education intervention Ethn Dis 2011; 21: 322-327.

36. Tol A, Shojaeezadeh D, Eslami A, et al. Evaluation of self-care practices and relative components among type 2 diabetes patients. $J$ Educ Health Promot 2012; 1: 9-13.

37. Ugwu E, Young E, Npozi M. Diabetes care knowledge and practice among primary care physicians in Southeast, Nigeria. BMC Fam Pract 2020; 21: 128.

38. Tewahido D, Berhane Y. Self-care practices among diabetes patients in Addis Ababa: a qualitative study. PLoS ONE 2017; 12(1): e0169062.

39. Leif G, Tiinamaija T, Nicola S, et al. The many faces of diabetes: a disease with increasing heterogeneity. Lancet 2014; 383(9922): 10841094.

Tables: 9

Figures: 2

References: 39

Received: 27.04 .2020

Reviewed: 10.07.2020

Accepted: 04.02.2021

Address for correspondence:

Busuyi Kolade Akinola, MD

Department of Human Anatomy

Federal University of Technology

Akure

Nigeria

Tel.: +234 8032073693

E-mail: bkakinola@futa.edu.ng 